\title{
ASYMPTOTIC EXPANSION FOR CLOSED GEODESICS IN HOMOLOGY CLASSES
}

\author{
DONGSHENG LIU ${ }^{1}$ \\ Department of Physics, Lancaster University, Lancaster, LA1 4YB, UK \\ e-mail:d.liu@lancaster.ac.uk
}

(Received 20 March, 2003; accepted 2 October 2003)

\begin{abstract}
In this paper we give a full asymptotic expansion for the number of closed geodesics in homology classes. Especially, we obtain formulae about the coefficients of error terms which depend on the homology class.
\end{abstract}

2000 Mathematics Subject Classification. 37C27, 37C30, 37D40, 35B40.

1. Introduction. Let $M$ be a compact Riemannian manifold with first Betti number $b>0$ equipped with negative sectional curvatures. There are a countable infinity closed geodesics on $M$, one in each non-zero conjugacy class in $\pi_{1} M$. The problem of obtaining asymptotic estimates on the number of closed geodesics has been studied by many authors. Let $\Gamma$ be the set of its closed geodesics. For $\gamma \in \Gamma$, let $l(\gamma)$ denote the length of $\gamma$, then

$$
\pi(T):=\#\{\gamma \in \Gamma: l(\gamma) \leq T\} \sim \frac{e^{h T}}{h T} \quad \text { as } \quad T \rightarrow \infty,
$$

where $h$ is the topological entropy of the geodesic flow on the unit-tangent bundle $S M$ [7].

We denote by $[\gamma] \in H_{1}(M, \mathbb{Z})$ the homology class of $\gamma$. For a fixed homology class $\alpha \in H_{1}(M, \mathbb{Z})$, it is known that

$$
\pi(T, \alpha):=\#\{\gamma \in \Gamma: l(\gamma) \leq T,[\gamma]=\alpha\} \sim c \frac{e^{T h}}{T^{b / 2+1}} \quad \text { as } \quad T \rightarrow \infty,
$$

for some constant $c>0,[5]$.

However the error terms of asymptotic expansion were not known until the work of Dolgopyat on Anosov flows [2], where he obtained strong results on the contractivity of transfer operators. These results led Anantharaman [1], Pollicott and Sharp [9] to find full expansions for $\pi(T, \alpha)$ and $\pi_{\delta}(T, \alpha)$ (the definition of $\pi_{\delta}(T, \alpha)$ follows).

In [1], by using direct approach based on Fourier-Laplace transform, Anantharaman obtained

$$
\begin{aligned}
\pi_{\delta}(T, \alpha): & =\#\{\gamma \in \Gamma:|l(\gamma)-T| \leq \delta,[\gamma]=\alpha\} \\
& =\frac{e^{T h}}{T^{b / 2+1}}\left(\sum_{n=0}^{N} \frac{c_{n}}{T^{n}}+O\left(T^{-(N+1)}\right)\right) \quad \text { as } \quad T \rightarrow \infty
\end{aligned}
$$

for all $N \in \mathbb{N}$, where the $c_{n}$ are constants.

$\overline{{ }^{1} \text { This work }}$ was done at the Department of Mathematics, University of Manchester. 
In fact, she obtained the result for Anosov flows whose stable and unstable characteristic foliations are of class $C^{1}$ and uniformly jointly non-integrable. Estimating the number of closed geodesics is a special case. Pollicott and Sharp [9] independently obtained the same results for $\pi(T, \alpha)$, but their expansion contained terms of the form $T^{-\frac{n}{2}}$ which should vanish when $n$ is odd.

However these authors did not describe how the constants $c_{n}$ depend on the homology class $\alpha$. In this article, we will consider how coefficients $c_{n}$ depend on $\alpha$ and give an explicit formula for $c_{n}$. Our main result is the following.

THEOREM. Let $M$ be a compact Riemannian manifold with first Betti number $b>0$ and with negative section curvatures. Furthermore suppose that either $\operatorname{dim} M=2$ or that the sectional curvatures lie between -4 and -1 . For a fixed $\alpha \in H_{1}(M, \mathbb{Z})$, we have

$$
\pi(T, \alpha)=\frac{e^{T h}}{T^{b / 2+1}}\left(c_{0}+\frac{c_{1}-\|\mid \alpha\| \|^{2}}{T}+O\left(T^{-2}\right)\right) \text { as } T \rightarrow \infty,
$$

where $h$ is the topological entropy of the geodesic flow on the unit-tangent bundle SM and III $\mid$ || is a norm on $H_{1}(M, \mathbb{R})$.

REMARKS.

1. The norm $\||\cdot|\|$ on $H_{1}(M, \mathbb{R})$ is defined by ||$x \mid \|=\langle x, A x\rangle$, where $A$ is a positive definite matrix which will be defined in section 6 .

2. We will obtain a more general form of the theorem, that is, we will give the formula of the coefficients of $T^{-n}$, for any $n \in \mathbb{N}$.

3. This method can be applied to an Anosov flow which is homologically full (i.e., every homology class contains at least one closed orbit) and whose stable and unstable characteristic foliations are $C^{1}$ and uniformly jointly non-integrable [6]. In fact, the pinching condition on the curvatures of $M$ ( sectional curvatures lie between -4 and $-1)$ is required to ensure that this condition holds.

4. After I obtained the results of this article, I learned that Motoko Kotani [4] had studied the same question and also showed how the coefficients $c_{n}(\alpha)$ depend on homology class $\alpha$. She followed the proof by Pollicott and Sharp in [9]. But my argument is by a more direct approach.

The analysis in this article is closely akin to that used by Anantharaman in [1], but we concentrate on how the coefficients depend on the homology class. In this article, the finite positive constants $c$ may vary at each occurrence.

2. Counting function. Let $M$ be the compact manifold with first Betti number $b>$ 0 and with negative sectional curvature. Let $S M$ denote the unit-tangent bundle. Given $(x, v) \in S M$, we can choose a unique unit speed geodesic $\gamma: \mathbb{R} \rightarrow M$ with $\gamma(0)=$ $x, \dot{\gamma}(0)=v$. We define the geodesic flow $\phi_{t}: S M \rightarrow S M$ by $\phi_{t}(x, v)=(\gamma(t), \dot{\gamma}(t))$ and let $h>0$ denote its topological entropy. There is a natural one-to-one correspondence between closed geodesics on $M$ and closed orbits for $\phi$. Let $\Gamma$ and $\tilde{\Gamma}$ denote respectively the set of closed geodesics on $M$ and the set of closed orbits for $\phi$. For $\gamma \in \Gamma$ we denote by $\tilde{\gamma}$ the corresponding closed orbit for $\phi$. Let $l(\gamma)$ and $l(\tilde{\gamma})$ denote the length of $\gamma$ and the least period of $\tilde{\gamma}$. It is known that $l(\gamma)=l(\tilde{\gamma})$.

For computational convenience, we fix an isomorphism between $H^{1}(M, \mathbb{R})$ and $\mathbb{R}^{b}$. This also gives an isomorphism between $H_{1}(M, \mathbb{Z}) /$ torsion and $\mathbb{Z}^{b}$. For simplicity, 
we shall consider manifolds $M$ whose first homology group is torsion free, i.e., $H_{1}(M, \mathbb{Z}) \cong \mathbb{Z}^{b}$. Otherwise, we can write $H_{1}(M, \mathbb{Z}) \cong \mathbb{Z}^{b} \oplus H$, where $H$ is the finite torsion subgroup. Our results will then only differ by a multiplicative constant.

For any closed geodesic $\gamma$ on $M$, there are $F_{i}: S M \rightarrow \mathbb{R}, i=1,2, \ldots, b$ such that

$$
[\gamma]=\left(\int_{\tilde{\gamma}} F_{1}, \int_{\tilde{\gamma}} F_{2}, \ldots, \int_{\tilde{\gamma}} F_{b}\right)=\int_{\tilde{\gamma}} F .
$$

Thus we may rewrite $\pi(T, \alpha)$ in the form

$$
\pi(T, \alpha)=\#\left\{\tilde{\gamma} \in \tilde{\Gamma}: l(\tilde{\gamma}) \leq T, \int_{\tilde{\gamma}} F=\alpha\right\} .
$$

We shall now concentrate on estimating $\pi(T, \alpha)$. For convenience, we shall abuse notation by writing $\gamma$ both for a closed geodesic on $M$ and for the corresponding closed orbit for the geodesic flow.

Let $G$ denote the closed subgroup of $\mathbb{R}^{b+1}$ generated by the vectors $\left(l(\gamma), \int_{\gamma} F\right)$ $(\gamma \in \Gamma)$. We have $G=\mathbb{R} \times \mathbb{Z}^{b}$. Let $M_{\phi}$ be the set of $\phi$-invariant probability measures on $S M$. For $u \in \mathbb{R}^{b}$, define function $\beta: \mathbb{R}^{b} \rightarrow \mathbb{R}$ by

$$
\beta(u)=\sup _{m \in M_{\phi}}\left\{h_{m}(\phi)+\left\langle u, \int F d m\right\rangle\right\} .
$$

$\beta(u)$ is an analytic function on $\mathbb{R}^{b}$ and has a positive definite Hessian at any point. It is well-known $\beta(0)=\sup _{m \in M_{\phi}}\left\{h_{m}\right\}=h$, the topological entropy of $\phi$ and $\nabla \beta(0)=0$. The function $\beta(u)$ can be continued analytically in a complex neighbourhood of 0 in $\mathbb{C}^{b}$.

In order to estimate $\pi(T, \alpha)$ we shall first consider the auxiliary function

$$
\pi_{g}(T, \alpha)=\sum_{[\gamma]=\alpha} g(l(\gamma)-T),
$$

where $g: \mathbb{R} \rightarrow \mathbb{R}^{+}$is a function on $\mathbb{R}$. First we assume that $g$ has a compact support and with $C^{3}$-regularity. Let $\hat{g}$ is the Fourier transform of $g$, by the Fourier Inversion Formula,

$$
\begin{aligned}
\pi_{g}(T, \alpha) & =\sum_{[\gamma]=\alpha} g(l(\gamma)-T) e^{\sigma(l(\gamma)-T)} e^{\sigma T} e^{-\sigma l(\gamma)} \\
& =\frac{1}{2 \pi} \sum_{\gamma \in \Gamma} \int_{\mathbb{R}} \int_{\mathbb{R}^{b} / \mathbb{Z}^{b}} \hat{g}(-i \sigma+t) e^{-i t(l(\gamma)-T)} e^{\sigma T} e^{-\sigma l(\gamma)} e^{2 \pi i\langle v,[\gamma]\rangle} e^{-2 \pi i\langle v, \alpha\rangle} d v d t \\
& =\frac{1}{2 \pi} \int_{\mathbb{R}} \int_{\mathbb{R}^{b} / \mathbb{Z}^{b}} Z(\sigma+i t, v) e^{\sigma T+i t T} \hat{g}(-i \sigma+t) e^{-2 \pi i\langle v, \alpha\rangle} d v d t,
\end{aligned}
$$

where we have defined

$$
Z(s, v)=Z(\sigma+i t, v)=\sum_{\gamma \in \Gamma} e^{-s l(\gamma)+2 \pi i\langle v,[\gamma]\rangle}=\sum_{\gamma \in \Gamma} e^{-s l(\gamma)+2 \pi i\left\langle v, \int_{\gamma} F\right\rangle}
$$

for $(s, v) \in \mathbb{C} \times \mathbb{R}^{b} / \mathbb{Z}^{b}$. It is well-known that when $\operatorname{Re} s=\sigma>\beta(0)=h, Z(s, v)$ is absolutely convergent. 
For the behaviour of $Z(s, v)$ in $\operatorname{Re} s<h$, we code the geodesic flow with suspended flow. Then we can determine the domain of $Z(s, v)$ by studying the norm of the transfer operator. Using the Dolgopyat's result that the spectrum of transfer operator is contracted when $\operatorname{Re} s>h-\epsilon$ and $|t| \rightarrow \infty$, we have the following proposition.

Proposition 1. [1] There exist $B>0, \epsilon_{1}>0, \epsilon_{2}>0, \epsilon_{3}>0$ and an open set $V_{0}, a$ neighbourhood of 0 in $\mathbb{R}^{b} / \mathbb{Z}^{b}$ such that

(1) $Z(s, v)$ is analytic if $\sigma>h-\epsilon_{1},|t|>B$ and in this domain we have $|Z(s, v)|<$ $c|t|$;

(2) $Z(s, v)+\log (s-\beta(i v))$ is analytic in $\left\{(s, v): \sigma>h-\epsilon_{2}, v \in V_{0}\right\}$;

(3) $Z(s, v)$ is analytic in $\left\{(s, v): v \notin \bar{V}_{0}, \sigma>h-\epsilon_{3}\right\}$.

3. Analysis of counting function. In this section we will analyse the counting function $\pi_{g}(T, \alpha)$. In what follows, let $g$ be of class $C^{3}$ with compact support. Let $\sigma>h$. Then

$$
\begin{aligned}
\pi_{g}(T, \alpha)= & \frac{1}{2 \pi} \int_{\mathbb{R} \times \mathbb{R}^{b} / \mathbb{Z}^{b}} Z(\sigma+i t, v) e^{(\sigma+i t) T} \hat{g}(-i \sigma+t) e^{-2 \pi i\langle v, \alpha\rangle} d t d v \\
= & \left.\frac{1}{2 \pi} \int_{V_{0}} e^{-2 \pi i\langle v, \alpha\rangle} d v \int_{\mathbb{R}} Z(\sigma+i t, v) e^{(\sigma+i t) T} \hat{g}(-i \sigma+t)\right) d t \\
& \left.+\frac{1}{2 \pi} \int_{\mathbb{R}^{b} / \mathbb{Z}^{b}-V_{0}} e^{-2 \pi i\langle v, \alpha\rangle} d v \int_{\mathbb{R}} Z(\sigma+i t, v) e^{(\sigma+i t) T} \hat{g}(-i \sigma+t)\right) d t .
\end{aligned}
$$

We shall consider the two integrals separately. First we consider the integral over $\mathbb{R}^{b} / \mathbb{Z}^{b}-V_{0}$. For $v \notin V_{0}$, we have the following estimate.

LeMma 1. For $v \notin V_{0}$ and for all $\sigma>h$, there exist $\epsilon>0$ such that

$$
\left|\int_{\mathbb{R}} Z(\sigma+i t, v) e^{(\sigma+i t) T} \hat{g}(-i \sigma+t) d t\right| \leq c\left\|g^{(3)}\right\|_{L^{1}} e^{T(h-\epsilon)} .
$$

Proof. Let $\sigma$ be fixed with $\sigma>h$. Let $\epsilon=\min \left\{\epsilon_{1}, \epsilon_{2}, \epsilon_{3}\right\}$, where $\epsilon_{1}, \epsilon_{2}, \epsilon_{3}$ are constants in Proposition 1. By part (3) of Proposition 1, $Z(s, v)$ is analytic in $\left\{(s, v): v \notin V_{0}, \operatorname{Re} s \geq h-\epsilon\right\}$. Using Cauchy's theorem,

$$
\int_{\Delta} Z(s, v) e^{s T} \hat{g}(-i s) d s=0,
$$

where $\Delta=\{\operatorname{Re} s=\sigma,|\operatorname{Im} s| \leq R\} \cup\{\operatorname{Re} s=h-\epsilon,|\operatorname{Im} s| \leq R\} \cup\{h-\epsilon \leq \operatorname{Re} s \leq \sigma$, $|\operatorname{Im} s|=R\}$.

Since $g$ is $C^{3}$ on $\mathbb{R}$ and has compact support, integrating by parts and only considering $g$ which has compact support contained in $(-\infty, 0]$, we obtain for $h-\epsilon \leq$ $\operatorname{Re} s \leq \sigma$,

$$
\hat{g}(-i s)=\left|\frac{1}{s^{3}} \int_{\mathbb{R}} g^{(3)}(y) e^{s y} d y\right| \leq c \frac{\left\|g^{(3)}\right\|_{L^{1}}}{|\operatorname{Im} s|^{3}},
$$

where $c$ is a constant which is independent of $g$. Using Proposition 1,

$$
\left|\int_{\{h-\epsilon \leq \operatorname{Re} s \leq \sigma,|\operatorname{Im} s|=R\}} Z(s, v) e^{s T} \hat{g}(-i s) d s\right| \leq c \operatorname{Re}^{\sigma T} c \frac{\left\|g^{(3)}\right\|_{L^{1}}}{R^{3}}(\sigma-(h-\epsilon)) \rightarrow 0
$$


as $R \rightarrow \infty$. This means that

$$
\int_{\{h-\epsilon \leq \operatorname{Re} s \leq \sigma,|\operatorname{Im} s|=R\}} Z(s, v) e^{s T} \hat{g}(-i s) d s \rightarrow 0 \quad \text { as } \quad R \rightarrow \infty .
$$

Since

$$
\int_{\mathbb{R}}\left|Z(\sigma+i t, v) e^{(\sigma+i t) T} \hat{g}(-i \sigma+t)\right| d t \leq c+2 \int_{B}^{+\infty} e^{\sigma T} c|t| c \frac{\left\|g^{(3)}\right\|_{L^{1}}}{|t|^{3}} d t<\infty,
$$

we have

$$
\lim _{R \rightarrow \infty} \int_{-R}^{R} Z(\sigma+i t, v) e^{(\sigma+i t) T} \hat{g}(-i \sigma+t) d t<\infty
$$

Similarly,

$$
\lim _{R \rightarrow \infty} \int_{-R}^{R} Z(h-\epsilon+i t, v) e^{(h-\epsilon+i t) T} \hat{g}(-i(h-\epsilon)+t) d t<\infty .
$$

Letting $R \rightarrow \infty$ in (1)and using (*), we have

$$
\begin{aligned}
& \left|\int_{\mathbb{R}} Z(\sigma+i t, v) e^{(\sigma+i t) T} \hat{g}(-i \sigma+t) d t\right| \\
& \quad \leq c+2 \int_{B}^{\infty} e^{(h-\epsilon) T} c|t| \cdot c \frac{\left\|g^{(3)}\right\|_{L^{1}}}{t^{3}} d t \leq c\left\|g^{(3)}\right\|_{L^{1}} e^{T(h-\epsilon)} .
\end{aligned}
$$

Now we consider the integral over $V_{0}$. We shall prove the following lemma.

LEMMA 2. Let $v \in V_{0}$, then for all $M \in \mathbb{N}$ and for all $\sigma>h$, we have

$$
\begin{aligned}
& \left|\int_{\mathbb{R}} Z(\sigma+i t, v) e^{(\sigma+i t) T} \hat{g}(-i \sigma+t) d t-2 \pi \sum_{j=0}^{M} \frac{1}{T^{j+1}} \frac{d^{j} \hat{g}}{d s^{j}}(-i \beta(i v)) e^{\beta(i v) T}\right| \\
& \leq c\left(\left\|g^{(3)}\right\|_{L^{1}}+\|g\|_{L^{1}}+\cdots+\left\|y^{M} g\right\|_{L^{1}}\right) e^{T(h-\epsilon)} \\
& \quad+\frac{c}{T^{M+1}}\left|\int_{C(\sigma)} \log (s-\beta(i v)) e^{s T} \frac{d^{M+1}}{d s^{M+1}} \hat{g}(-i s) d s\right|
\end{aligned}
$$

where $C(\sigma)$ will be defined later.

Proof. When $v \in V_{0}, \sigma$ is fixed with $\sigma>h$. For $2 B<R \in \mathbb{R}^{+}$, let

$C_{0}=\{\operatorname{Re} s=h-\epsilon,-2 B \leq \operatorname{Im} s \leq 2 B\} ; \quad C_{1}=\{\operatorname{Im} s=-2 B, h-\epsilon \leq \operatorname{Re} s \leq \sigma\} ;$

$C_{2}=\{\operatorname{Re} s=\sigma,-2 B \leq \operatorname{Im} s \leq 2 B\} ; \quad C_{3}=\{\operatorname{Im} s=2 B, h-\epsilon \leq \operatorname{Re} s \leq \sigma\} ;$

$C_{4}^{+}=\{\operatorname{Re} s=h-\epsilon, 2 B \leq \operatorname{Im} s \leq R\} ; \quad C_{4}^{-}=\{\operatorname{Re} s=h-\epsilon,-R \leq \operatorname{Im} s \leq-2 B\} ;$

$C_{5}^{+}=\{\operatorname{Re} s=\sigma, 2 B \leq \operatorname{Im} s \leq R\} ; \quad C_{5}^{-}=\{\operatorname{Re} s=\sigma,-R \leq \operatorname{Im} s \leq-2 B\} ;$

$C_{R}^{+}=\{h-\epsilon \leq \operatorname{Re} s \leq \sigma, \operatorname{Im} s=R\} ; \quad C_{R}^{-}=\{h-\epsilon \leq \operatorname{Re} s \leq \sigma, \operatorname{Im} s=-R\}$.

By part (1) of Proposition 1, Z(s,v) is analytic in $\operatorname{Re} s>h-\epsilon,|\operatorname{Im} s|>B$,

$$
\begin{aligned}
& \int_{\left\{C_{3} \cup C_{5}^{+} \cup C_{R}^{+} \cup C_{4}^{+}\right\}} Z(s, v) e^{s T} \hat{g}(-i s) d s=0, \\
& \int_{\left\{C_{1} \cup C_{4}^{-} \cup C_{R}^{-} \cup C_{5}^{-}\right\}} Z(s, v) e^{s T} \hat{g}(-i s) d s=0 .
\end{aligned}
$$


By part (2) of Proposition 1, we have

$$
\int_{-\left\{C_{0} \cup C_{3} \cup C_{2} \cup C_{1}\right\}}(Z(s, v)+\log (s-\beta(i v))) e^{s T} \hat{g}(-i s) d s=0
$$

where the three contours are counterclockwise and $-C_{i}$ means that the orientation of the path is reversed.

Letting $R \rightarrow \infty$ in (2) and using (*), we have

$$
\int_{\{\operatorname{Re} s=\sigma, 2 B \leq \operatorname{Im} s \leq \infty\}} Z(s, v) e^{s T} \hat{g}(-i s) d s=\int_{-C_{3} \cup\{\operatorname{Re} s=h-\epsilon, 2 B \leq \operatorname{Im} s \leq \infty\}} Z(s, v) e^{s T} \hat{g}(-i s) d s .
$$

Letting $R \rightarrow \infty$ in (3), we have

$$
\begin{aligned}
& \int_{\{\operatorname{Re} s=\sigma,-\infty \leq \operatorname{Im} s \leq-2 B\}} Z(s, v) e^{s T} \hat{g}(-i s) d s \\
& \quad=\int_{-C_{1} \cup\{\operatorname{Re} s=h-\epsilon,-\infty \leq \operatorname{Im} s \leq-2 B\}} Z(s, v) e^{s T} \hat{g}(-i s) d s .
\end{aligned}
$$

From (4), we have

$$
\begin{aligned}
\int_{-C_{2}} Z(s, v) e^{s T} \hat{g}(-i s) d s= & \int_{C_{1} \cup C_{2} \cup C_{3}} \log (s-\beta(i v)) e^{s T} \hat{g}(-i s) d s \\
& +\int_{C_{0}}(Z(s, v)+\log (s-\beta(i v))) e^{s T} \hat{g}(-i s) d s \\
& +\int_{C_{1} \cup C_{3}} Z(s, v) e^{s T} \hat{g}(-i s) d s
\end{aligned}
$$

Let $C(\sigma)=C_{1} \cup C_{2} \cup C_{3}$. Adding three identities (5), (6), (7) we obtain

$$
\begin{aligned}
& \left|\int_{\mathbb{R}} Z(\sigma+i t, v) e^{(\sigma+i t) T} \hat{g}(-i \sigma+t) d t+i \int_{C(\sigma)} \log (s-\beta(i v)) \hat{g}(-i s) e^{s T} d s\right| \\
& \leq\left|\int_{\{\operatorname{Re} s=h-\epsilon,|\operatorname{Im} s|>2 B\}} Z(s, v) e^{s T} \hat{g}(-i s) d s\right| \\
& \quad+\left|\int_{C_{0}}(Z(s, v)+\log (s-\beta(i v))) e^{s T} \hat{g}(-i s) d s\right| \\
& \leq 2 e^{T(h-\epsilon)} \int_{2 B}^{\infty} c|t| \frac{\left\|g^{(3)}\right\|_{L^{1}}}{t^{3}} d t+c e^{T(h-\epsilon)} \leq c\left\|g^{(3)}\right\|_{L^{1}} e^{T(h-\epsilon)} .
\end{aligned}
$$

Integrating by parts, we have

$$
\begin{gathered}
\mid i \int_{C(\sigma)} \log (s-\beta(i v)) \hat{g}(-i s) e^{s T} d s+\frac{i}{T} \int_{C(\sigma)} \frac{\hat{g}(-i s)}{s-\beta(i v)} e^{s T} d s \\
\quad+\frac{i}{T} \int_{C(\sigma)} \log (s-\beta(i v)) \frac{d \hat{g}}{d s}(-i s) e^{s T} d s \mid \leq c\|g\|_{L^{1}} e^{T(h-\epsilon)}
\end{gathered}
$$


By using the Residue Formula,

$$
\begin{aligned}
\left|\int_{C(\sigma)} \frac{\hat{g}(-i s)}{s-\beta(i v)} e^{s T} d s+2 \pi i \hat{g}(-i \beta(i v)) e^{\beta(i v) T}\right| & =\left|\int_{C_{0}} \frac{\hat{g}(-i s)}{s-\beta(i v)} e^{s T} d s\right| \\
& \leq \int_{-2 B}^{2 B}\left|\frac{\hat{g}(t-i(h-\epsilon))}{h-\epsilon+i t-\beta(i v)} e^{((h-\epsilon)+i t) T} d t\right| \\
& \leq c\|g\|_{L^{1}} e^{T(h-\epsilon)} .
\end{aligned}
$$

So we have obtained

$$
\begin{aligned}
& \left|\int_{\mathbb{R}} Z(\sigma+i t, v) e^{(\sigma+i t) T} \hat{g}(-i \sigma+t) d t-2 \pi \frac{1}{T} \hat{g}(-i \beta(i v)) e^{\beta(i v) T}\right| \\
& \quad \leq c\left(\left\|g^{(3)}\right\|_{L^{1}}+\|g\|_{L^{1}}\right) e^{T(h-\epsilon)}+\frac{c}{T}\left|\int_{C(\sigma)} \log (s-\beta(i v)) e^{s T} \frac{d \hat{g}(-i s)}{d s} d s\right|
\end{aligned}
$$

We iterate the preceding operation $M+1$ times, and note

$$
\left|\frac{d^{k} \hat{g}(-i s)}{d s^{k}}\right|=\left|\int_{\mathbb{R}} y^{k} g(y) e^{s y} d y\right| \leq c\left\|y^{k} g\right\|_{L^{1}} .
$$

We have

$$
\begin{aligned}
& \left|\int_{\mathbb{R}} Z(\sigma+i t, v) e^{(\sigma+i t) T} \hat{g}(-i \sigma+t) d t-2 \pi \sum_{j=0}^{M} \frac{1}{T^{j+1}} \frac{d^{j} \hat{g}}{d s^{j}}(-i \beta(i v)) e^{\beta(i v) T}\right| \\
& \leq c\left(\left\|g^{(3)}\right\|_{L^{1}}+\|g\|_{L^{1}}+\cdots+\left\|y^{M} g\right\|_{L^{1}}\right) e^{T(h-\epsilon)} \\
& \quad+\frac{c}{T^{M+1}}\left|\int_{C(\sigma)} \log (s-\beta(i v)) e^{s T} \frac{d^{M+1}}{d s^{M+1}} \hat{g}(-i s) d s\right| .
\end{aligned}
$$

Using the Dominated Convergence Theorem, we have

$$
\begin{gathered}
\lim _{\sigma \rightarrow h} \int_{V_{0}} e^{-2 \pi i\langle v, \alpha\rangle} d v \int_{C(\sigma)} \log (s-\beta(i v)) e^{s T} \frac{d^{M+1}}{d s^{M+1}} \hat{g}(-i s) d s \\
\quad=\int_{V_{0}} e^{-2 \pi i\langle v, \alpha\rangle} d v \int_{C(h)} \log (s-\beta(i v)) e^{s T} \frac{d^{M+1}}{d s^{M+1}} \hat{g}(-i s) d s .
\end{gathered}
$$

By Lemma 1 and Lemma 2, we can prove the following proposition.

Proposition 2. Let $g$ be class $C^{3}$ with compact support. For all $M \geq 1$, we have

$$
\begin{aligned}
& \left|\pi_{g}(T, \alpha)-\sum_{j=0}^{M} \frac{1}{T^{j+1}} \int_{V_{0}} e^{-2 \pi i\langle v, \alpha\rangle} \frac{d^{j} \hat{g}}{d s^{j}}(-i \beta(i v)) e^{\beta(i v) T} d v\right| \\
& \quad \leq c \frac{\left\|y^{M+1} g\right\|_{L^{1}}}{T^{M+1}} e^{T h}+c\left(\left\|g^{(3)}\right\|_{L^{1}}+\|g\|_{L^{1}}+\cdots+\left\|y^{M} g\right\|_{L^{1}}\right) e^{T(h-\epsilon)} .
\end{aligned}
$$


4. Further calculation of counting function. By Proposition 2, in order to obtain the estimate of $\pi_{g}(T, \alpha)$, we only need to estimate

$$
\int_{V_{0}} \frac{d^{j} \hat{g}}{d s^{j}}(-i \beta(i v)) e^{T \beta(i v)} e^{-2 \pi i\langle v, \alpha\rangle} d v .
$$

We first have the following lemma.

LEMMA 3. There exist polynomials $f_{j}^{(k)}(i v)$ in $i v_{1}, \ldots, i v_{b}$ such that the total exponent of each term has the same parity as $k$ and such that

$$
\begin{aligned}
& \mid \int_{V_{0}} \frac{d^{j} \hat{g}}{d s^{j}}(-i \beta(i v)) e^{T \beta(i v)-2 \pi i\langle v, \alpha\rangle} d v \\
& \quad-\frac{e^{T h}}{T^{b / 2}} \sum_{k=0}^{2 N+1} \frac{1}{T^{k / 2}} \int_{\|v\| \leq \sqrt{T} \rho} e^{-\frac{1}{2} \beta^{\prime \prime}(0)(v, v)-2 \pi i\left\langle\frac{v}{\sqrt{T}}, \alpha\right\rangle} f_{j}^{(k)}(i v) d v \mid \\
& \quad \leq c \sup _{n \leq 2 N+2}\left\|y^{j+n} g\right\|_{L^{1}} \frac{e^{T h}}{T^{N+\frac{b}{2}+1}}
\end{aligned}
$$

for some small $\rho$.

REMARKS.

(1) Here $\|\cdot\|$ denotes the 2-norm, i.e., $\|v\|=\left(\sum_{i=1}^{b} v_{i}^{2}\right)^{1 / 2}$,

(2) The proof of this lemma is same as that in [1]. We denote $\frac{d^{j} \hat{g}}{d s^{j}}(-i \beta(i v))$ by $\bar{g}_{j}(i v)$, then expand $\beta\left(\frac{i v}{\sqrt{T}}\right)$ and $\bar{g}_{j}\left(\frac{i v}{\sqrt{T}}\right)$ in the neighbourhood of 0 by

$$
\begin{aligned}
\beta\left(\frac{i v}{\sqrt{ } T}\right) & =\beta(0)+\nabla \beta(0) \cdot i v+\frac{1}{2} \beta^{\prime \prime}(0)(i v, i v)+T R_{2 N+3} \\
& =h-\frac{1}{2} \beta^{\prime \prime}(0)(v, v)+T R_{2 N+3}
\end{aligned}
$$

and

$$
\bar{g}_{j}(i v / \sqrt{T})=\sum_{l=0}^{2 N+1} \frac{\bar{g}_{j}^{(l)}(0)}{l !} \cdot(i v)^{l} T^{-l / 2}+Y_{N}(i v / \sqrt{T}) .
$$

(3) By the first condition, we mean that $f_{j}^{(k)}(i v)$ can be written in the form

$$
f_{j}^{(k)}(i v)=\sum a_{l_{1}, l_{2}, \ldots, l_{b}}\left(i v_{1}\right)^{l_{1}}\left(i v_{2}\right)^{l_{2}} \cdots\left(i v_{b}\right)^{l_{b}} .
$$

In particular,

$$
\begin{aligned}
f_{j}^{(0)}(i v)= & \bar{g}_{j}^{(0)}(0)=\left.\frac{d^{j}}{d s^{j}} \hat{g}(-i s)\right|_{s=h} \\
f_{j}^{(1)}(i v)= & \frac{1}{6} \bar{g}_{j}^{(0)}(0) \beta^{(3)}(0) \cdot(i v)^{3}+\bar{g}_{j}^{(1)}(0) \cdot(i v) ; \\
f_{j}^{(2)}(i v)= & \frac{1}{72} \bar{g}_{j}^{(0)}(0)\left(2\left(\beta^{(3)}(0) \cdot(i v)^{3}\right)^{2}+3 \beta^{(4)}(0) \cdot(i v)^{4}\right) \\
& +\frac{1}{6} \bar{g}_{j}^{(1)}(0) \cdot(i v) \beta^{(3)}(0) \cdot(i v)^{3}+\frac{1}{2} \bar{g}_{j}^{(2)}(0) \cdot(i v)^{2} .
\end{aligned}
$$


(4) We can see that $f_{j}^{(0)}(i v)$ are constants and $f_{0}^{(0)}(i v)>0$, since

$$
\begin{aligned}
f_{j}^{(0)}(i v) & =\left.\frac{d^{j}}{d s^{j}} \hat{g}(-i s)\right|_{s=h}=\left.\frac{d^{j}}{d s^{j}} \int_{\mathbb{R}} g(y) e^{s y} d y\right|_{s=h} \\
& =\left.\int_{\mathbb{R}} y^{j} g(y) e^{s y} d y\right|_{s=h}=\int_{\mathbb{R}} y^{j} g(y) e^{h y} d y .
\end{aligned}
$$

Taking $M=N+b+2$ in (8), we have proved the following proposition.

Proposition 3. For any $N \geq 1$, we have

$$
\begin{aligned}
& \left|\pi_{g}(T, \alpha)-\frac{e^{T h}}{T^{b / 2+1}} \sum_{j=0}^{N+b+2} \frac{1}{T^{j}} \sum_{k=0}^{2 N+1} \frac{1}{T^{k / 2}} \int_{\|v\| \leq \sqrt{T} \rho} e^{-\frac{1}{2} \beta^{\prime \prime}(0)(v, v)} e^{-2 \pi i\langle\alpha, v / \sqrt{T}\rangle} f_{j}^{(k)}(i v) d v\right| \\
& \quad \leq c \sup _{n \leq 4 N+b+2}\left\|y^{n} g\right\|_{L^{1}} \frac{e^{T h}}{T^{N+\frac{b}{2}+2}}+c\left(\left\|g^{(3)}\right\|_{L^{1}}+\|g\|_{L^{1}}+\cdots+\left\|y^{N+b+2} g\right\|_{L^{1}}\right) e^{T(h-\epsilon)},
\end{aligned}
$$

where $f_{j}^{(k)}(i v)$ in $i v_{1}, \ldots, i v_{b}$ are polynomials such that the total exponent of each term has the same parity as $k$.

5. Coefficients of error terms of $\pi_{g}(T, \alpha)$. In this section, we will give the asymptotic formula for $\pi_{g}(T, \alpha)$ with error terms. We also give the explicit expression of coefficients of error terms.

By the Proposition 3, we need to estimate

$$
\sum_{k=0}^{2 N+1} \frac{1}{T^{k / 2}} \int_{\|v\| \leq \sqrt{T} \rho} e^{-\frac{1}{2} \beta^{\prime \prime}(0)(v, v)} e^{-2 \pi i\langle\alpha, v / \sqrt{T}\rangle} f_{j}^{(k)}(i v) d v
$$

where $f_{j}^{(k)}(i v)$ are polynomials in $i v_{1}, \ldots, i v_{b}$ such that the total exponent of each term in $f_{j}^{(k)}$ and $k$ have the same parity. That is, we can write

$$
f_{j}^{(k)}(i v)=\sum \bar{b}_{l_{1}, l_{2}, \ldots, l_{b}}\left(i v_{1}\right)^{l_{1}}\left(i v_{2}\right)^{l_{2}} \cdots\left(i v_{b}\right)^{l_{b}}
$$

where $l_{1}+l_{2}+\cdots+l_{b}$ and $k$ have the same parity.

We first prove the following proposition.

Proposition 4.

$$
\begin{aligned}
& \sum_{k=0}^{2 N+1} \frac{1}{T^{k / 2}} \int_{\|v\| \leq \sqrt{T} \rho} e^{-\frac{1}{2} \beta^{\prime \prime}(0)(v, v)} e^{-2 \pi i\langle\alpha, v / \sqrt{T}\rangle} f_{j}^{(k)}(i v) d v \\
& \quad=\sum_{k=0}^{2 N+1} \frac{1}{T^{k / 2}} \int_{\mathbb{R}^{b}} e^{-\frac{1}{2} \beta^{\prime \prime}(0)(v, v)} s_{j}^{(k)}(\alpha, i v) d v+O\left(\sup _{n \leq 4 N+b+2}\left\|y^{n} g\right\|_{L^{1}} T^{-(N+1)}\right),
\end{aligned}
$$

where

$$
s_{j}^{(k)}(\alpha, i v)=\sum_{l=0}^{k}(-1)^{k-l} \frac{f_{j}^{(l)}(i v)\langle\alpha, 2 \pi i v\rangle^{k-l}}{(k-l) !}
$$


are polynomials in $i v_{1}, \ldots, i v_{b}$ and we still have that the total exponent of each term in $s_{j}^{(k)}(\alpha, v)$ has the same parity as $k$.

Proof. We expand $e^{-2 \pi i\langle\alpha, v / \sqrt{T}\rangle}$ in a neighborhood of 0 .

$$
\begin{aligned}
e^{-2 \pi i\langle\alpha, v / \sqrt{T}\rangle}= & 1-\frac{\langle\alpha, 2 \pi i v\rangle}{\sqrt{T}}+\frac{\langle\alpha, 2 \pi i v\rangle^{2}}{2 T}-\frac{\langle\alpha, 2 \pi i v\rangle^{3}}{3 ! T^{3 / 2}} \\
& +\cdots-\frac{\langle\alpha, 2 \pi i v\rangle^{2 N+1}}{(2 N+1) ! T^{N / 2+1}}+Z_{N}(i v / \sqrt{T}),
\end{aligned}
$$

where $\left|Z_{N}(i v / \sqrt{T})\right| \leq c \frac{\|v\|^{2 N+2}}{T^{N+1}}$. Let

$$
s_{j}^{(k)}(\alpha, i v)=\sum_{l=0}^{k}(-1)^{k-l} \frac{f_{j}^{(l)}(i v)\langle\alpha, 2 \pi i v\rangle^{k-l}}{(k-l) !}
$$

then

$$
\begin{aligned}
& \sum_{k=0}^{2 N+1} \frac{1}{T^{k / 2}} \int_{\|v\| \leq \sqrt{T} \rho} e^{-\frac{1}{2} \beta^{\prime \prime}(0)(v, v)} e^{-2 \pi i\langle\alpha, v / \sqrt{T}\rangle} f_{j}^{(k)}(i v) d v \\
& \quad=\sum_{k=0}^{2 N+1} \frac{1}{T^{k / 2}} \int_{\|v\| \leq \sqrt{T} \rho} e^{-\frac{1}{2} \beta^{\prime \prime}(0)(v, v)} s_{j}^{(k)}(\alpha, i v) d v+O\left(\sup _{n \leq 4 N+b+2}\left\|y^{n} g\right\|_{L^{1}} T^{-(N+1)}\right) .
\end{aligned}
$$

For $T$ is sufficiently large, for all $m \in \mathbb{N}$, we have

$$
\begin{aligned}
\int_{\|v\|>\sqrt{T} \rho} e^{-\frac{1}{2} \beta^{\prime \prime}(0)(v, v)}\|v\|^{m} d v & \leq \int_{\|v\|>\sqrt{T} \rho} e^{-\epsilon^{\prime}\|v\|^{2}}\left|v_{1} v_{2} \cdot v_{d}\right| d v \\
& \leq \prod_{i=1}^{d} \int_{\sqrt{T} \rho}^{\infty} e^{-\epsilon^{\prime} v_{i}^{2}} v_{i} d v_{i} \leq c e^{-\epsilon^{\prime} T}
\end{aligned}
$$

for some $\epsilon^{\prime}>0$. So we have

$$
\begin{aligned}
& \mid \sum_{k=0}^{2 N+1} \frac{1}{T^{k / 2}} \int_{\|v\| \leq \sqrt{T} \rho} e^{-\frac{1}{2} \beta^{\prime \prime}(0)(v, v)} e^{-2 \pi i\langle\alpha, v / \sqrt{T}\rangle} f_{j}^{(k)}(i v) d v \\
& \quad-\sum_{k=0}^{2 N+1} \frac{1}{T^{k / 2}} \int_{\mathbb{R}^{b}} e^{-\frac{1}{2} \beta^{\prime \prime}(0)(v, v)} s_{j}^{(k)}(\alpha, i v) d v \mid \\
& \quad \leq c \sup _{n \leq 4 N+b+2}\left\|y^{n} g\right\|_{L^{1}}\left(T^{-(N+1)}+e^{-\epsilon^{\prime} T}\right) .
\end{aligned}
$$

In the following lemma, we will see that the coefficients of $T^{-\frac{k}{2}}$ vanish when $k$ is odd.

LEMMA 4. If $k$ is odd, then

$$
\int_{\mathbb{R}^{b}} e^{-\frac{1}{2} \beta^{\prime \prime}(0)(v, v)} s_{j}^{(k)}(\alpha, i v) d v=0 .
$$


Proof.

$$
\begin{aligned}
s_{j}^{(k)}(\alpha, i v) & =\sum_{l=0}^{k}(-1)^{k-l} \frac{f_{j}^{(l)}(i v)\langle\alpha, 2 \pi i v\rangle^{k-l}}{(k-l) !} \\
& =\sum_{l=0}^{k}(-1)^{k-l} f_{j}^{(l)}(i v)(2 \pi i)^{k-l}\left(\sum_{i=1}^{b} \alpha_{i} v_{i}\right)^{k-l} \\
& :=\sum a_{l_{1}, l_{2}, \ldots, l_{b}}(\alpha)\left(i v_{1}\right)^{l_{1}}\left(i v_{2}\right)^{l_{2}} \ldots\left(i v_{b}\right)^{l_{b}},
\end{aligned}
$$

where $l_{1}+l_{2}+\cdots+l_{b}$ is odd and $a_{l_{1}, L_{2}, \ldots, l_{b}}(\alpha)$ are constants which depend on $\alpha$ and $g$. Let $v^{\prime}=-v$, i.e., $\left(v_{1}^{\prime}, v_{2}^{\prime}, \ldots, v_{b}^{\prime}\right)=\left(-v_{1},-v_{2}, \ldots,-v_{b}\right)$; then

$$
\begin{aligned}
& \int_{\mathbb{R}^{b}} e^{-\frac{1}{2} \beta^{\prime \prime}(0)(v, v)} s_{j}^{(k)}(\alpha, i v) d v \\
& =\int_{\mathbb{R}^{b}} e^{-\frac{1}{2} \beta^{\prime \prime}(0)(v, v)} \sum a_{l_{1}, l_{2}, \ldots, l_{b}}\left(i v_{1}\right)^{l_{1}}\left(i v_{2}\right)^{l_{2}} \cdots\left(i v_{b}\right)^{l_{b}} d v_{1} d v_{2} \cdots d v_{b} \\
& =-\int_{\mathbb{R}^{b}} e^{-\frac{1}{2} \beta^{\prime \prime}(0)\left(v^{\prime}, v^{\prime}\right)} s_{j}^{(k)}\left(\alpha, i v^{\prime}\right) d v^{\prime} .
\end{aligned}
$$

Thus

$$
\int_{\mathbb{R}^{b}} e^{-\frac{1}{2} \beta^{\prime \prime}(0)(v, v)} s_{j}^{(k)}(\alpha, i v) d v=0 .
$$

By Lemma 4, for $k$ odd the coefficient of $T^{-\frac{k}{2}}$ vanish. So we only need to calculate coefficients when $k$ is even. Let $b_{j}^{(k)}(\alpha)$ be the coefficient of $T^{k}$ in Proposition 4; if we write $\alpha=\left(\alpha_{1}, \alpha_{2}, \ldots, \alpha_{b}\right)$ then

$$
\begin{aligned}
b_{j}^{(k)}(\alpha) & =\int_{\mathbb{R}^{b}} e^{-\frac{1}{2} \beta^{\prime \prime}(0)(v, v)} s_{j}^{(2 k)}(\alpha, i v) d v \\
& =\int_{\mathbb{R}^{b}} e^{-\frac{1}{2} \beta^{\prime \prime}(0)(v, v)} \sum_{l=0}^{2 k}(-1)^{l} \frac{f_{j}^{(l)}(i v)\langle\alpha, 2 \pi i v\rangle^{2 k-l}}{(2 k-l) !} d v \\
& =\sum_{l_{1}+l_{2}+\cdots+l_{b}=0}^{2 k} b_{l_{1} l_{2} \ldots l_{b}}^{(j)} \alpha_{1}^{l_{1}} \alpha_{2}^{l_{2}} \cdots \alpha_{b}^{l_{b}},
\end{aligned}
$$

where $b_{l_{1} l_{2} \ldots l_{b}}^{(j)}$ are constants. More precisely,

$$
\begin{aligned}
b_{l_{1} l_{2} \ldots l_{b}}^{(j)}= & \frac{\left(l_{1}+l_{2}+\cdots+l_{b}\right) !}{l_{1} ! l_{2} ! \ldots l_{b} !} \int_{\mathbb{R}^{b}} e^{-\frac{1}{2} \beta^{\prime \prime}(0)(v, v)}(2 \pi i)^{l_{1}+l_{2}+\cdots+l_{b}} \\
& \times v_{1}^{l_{1}} v_{2}^{l_{2}} \ldots v_{b}^{l_{b}} f_{j}^{\left(2 k-\left(l_{1}+l_{2}+\cdots+l_{b}\right)\right)}(i v) d v .
\end{aligned}
$$

Then we have proved the following Proposition. 
Proposition 5.

$$
\begin{aligned}
& \left|\sum_{k=0}^{2 N+1} \frac{1}{T^{k / 2}} \int_{\|v\| \leq \sqrt{T} \rho} e^{-\frac{1}{2} \beta^{\prime \prime}(0)(v, v)} e^{-2 \pi i\langle\alpha, v / \sqrt{T}\rangle} f_{j}^{(k)}(i v) d v-\sum_{k=0}^{N} \frac{b_{j}^{(k)}(\alpha)}{T^{k}}\right| \\
& \quad \leq c \sup _{n \leq 4 N+b+2}\left\|y^{n} g\right\|_{L^{1}}\left(T^{-(N+1)}+e^{-\epsilon^{\prime} T}\right),
\end{aligned}
$$

where $b_{j}^{(k)}(\alpha)=\sum_{l_{1}+l_{2}+\cdots+l_{b}=0}^{2 k} b_{l_{1} l_{2} \ldots l_{b}}^{(j)} \alpha_{1}^{l_{1}} \alpha_{2}^{l_{2}} \ldots \alpha_{b}^{l_{b}}$ are polynomials in $\alpha_{1}, \alpha_{2}, \ldots, \alpha_{b}$ and the degree of $b_{j}^{(k)}$ is $2 k$ and $b_{l_{1} l_{2} \ldots l_{b}}^{(j)}$ are constants which depend on $g$.

In order to obtain $\pi_{g}(T, \alpha)$, we shall use Proposition 4 and Proposition 5. Let

$$
\begin{aligned}
c_{n}(\alpha) & =\sum_{i=0}^{n} b_{i}^{(n-i)}(\alpha)=\sum_{i=0}^{n}\left(\sum_{l_{1}+l_{2}+\cdots+l_{b}=0}^{2(n-i)} b_{l_{1} l_{2} \ldots l_{b}}^{(j)} \alpha_{1}^{l_{1}} \alpha_{2}^{l_{2}} \ldots \alpha_{b}^{l_{b}}\right) \\
& :=\sum_{l_{1}+l_{2}+\cdots+l_{b}=0}^{2 n} c_{l_{1} l_{2} \ldots l_{b}} \alpha_{1}^{l_{1}} \alpha_{2}^{l_{2}} \ldots \alpha_{b}^{l_{b}},
\end{aligned}
$$

where $c_{l_{1} l_{2} \ldots l_{b}}$ are constants and

$$
c_{0}(\alpha)=b_{0}^{(0)}=f_{0}^{(0)} \int_{\mathbb{R}^{b}} e^{-\frac{1}{2} \beta^{\prime \prime}(0)(v, v)} d v=\frac{(2 \pi)^{b / 2}}{\sqrt{\left|\operatorname{det} \beta^{\prime \prime}(0)\right|}} \hat{g}(-i h)>0,
$$

so $c_{0}(\alpha)$ is independent of $\alpha$. On the other hand, for $n \geq 1, c_{n}(\alpha)$ is polynomial in $\alpha_{1}, \ldots, \alpha_{b}$ whose degree is $2 n$ by Proposition 5. From the expression of $b_{j}^{(k)}(\alpha)$, we have

$$
\begin{aligned}
c_{n}(\alpha) & \sim \int_{\mathbb{R}^{b}} e^{-\frac{1}{2} \beta^{\prime \prime}(0)(v, v)} f_{0}^{(0)}(i v) \frac{\langle\alpha, 2 \pi i v\rangle^{2 n}}{(2 n) !} d v \\
& =(2 \pi i)^{2 n} \frac{f_{0}^{(0)}}{(2 n) !} \int_{\mathbb{R}^{b}} e^{-\frac{1}{2} \beta^{\prime \prime}(0)(v, v)}\langle v, \alpha\rangle^{2 n} d v \\
& \sim(-1)^{n} c_{g}\|\alpha\|^{2 n} \quad\left(c_{g}>0 \text { and } c_{g} \text { is dependent on } g\right),
\end{aligned}
$$

where $A \sim B$ means that $\lim _{\|\alpha\| \rightarrow \infty} A / B=1$.

We have now obtained the following theorem.

THEOREM 1. Let $g$ be of class $C^{3}$ with compact support. Then for all $N \geq 1$, we have

$$
\pi_{g}(T, \alpha)=\frac{e^{T h}}{T^{b / 2+1}}\left(\sum_{n=0}^{N} \frac{c_{n, g}(\alpha)}{T^{n}}+O\left(T^{-(N+1)}\right)\right),
$$

where $c_{n, g}(\alpha), n=0,1, \ldots, N$ are constants with $c_{0, g}>0$ independent of $\alpha$ but dependent on $g$ and for $n \geq 1, \quad c_{n, g}(\alpha)=\sum_{l_{1}+l_{2}+\cdots+l_{b}=0}^{2 n} c_{l_{1} l_{2} \ldots l_{b}} \alpha_{1}^{l_{1}} \alpha_{2}^{l_{2}} \ldots \alpha_{b}^{l_{b}}$ is a polynomial in $\alpha_{1}, \alpha_{2}, \ldots, \alpha_{b}$ whose degree is $2 n$ with $c_{l_{1} l_{2} \ldots l_{b}}$ constants depending on $g$. Furthermore, $c_{n, g}(\alpha) \sim(-1)^{n} c_{g}\|\alpha\|^{2 n}$, as $\|\alpha\| \rightarrow \infty$. 


\section{REMARKS.}

(1) The explicit formulae for $c_{0, g}$ and $c_{1, g}(\alpha)$ are following.

$$
\begin{aligned}
c_{0, g} & =b_{0}^{(0)}=\frac{(2 \pi)^{b / 2}}{\sqrt{\left|\operatorname{det} \beta^{\prime \prime}(0)\right|}} \hat{g}(-i h)>0 ; \\
c_{1, g}(\alpha) & =b_{0}^{(1)}+b_{1}^{(0)} \\
& =\int_{\mathbb{R}^{b}} e^{-\frac{1}{2} \beta^{\prime \prime}(0)(v, v)}\left(\frac{1}{2} f_{0}^{(0)}(i v)\langle\alpha, 2 \pi i v\rangle^{2}-f_{0}^{(1)}(i v)\langle\alpha, 2 \pi i v\rangle+f_{0}^{(2)}(i v)+f_{1}^{(0)}(i v)\right) d v .
\end{aligned}
$$

(2) The term $O\left(T^{-(N+1)}\right)$ can be bounded by $C \sup _{n \leq 4 N+b+2}\left\|y^{n} g\right\|_{L^{1}} T^{-(N+1)}+$ $c\left\|g^{(3)}\right\|_{L^{1}} e^{-\epsilon T}$.

6. The proof of main results. In this section, we use approximation to estimate $\pi(T, \alpha)$.

For $N \in \mathbb{N}$, let $g=\chi_{\left[-T^{\frac{1}{4 N+b+3}}, 0\right]}$. For all $T$ we take $g_{T}^{-}$and $g_{T}^{+}$of class $C^{3}$ with compact supports such that

(1) $g_{T}^{-} \leq g \leq g_{T}^{+}$;

(2) $\left\|g_{T}^{+}\right\|_{\infty} \leq 2$ and $\left\|g_{T}^{-}\right\|_{\infty} \leq 2$;

(3) for $0 \leq n \leq 4 N+b+2,\left\|y^{n} g_{T}^{ \pm}\right\|_{L^{1}} \leq c\left\|y^{n} g\right\|_{L^{1}}$;

(4) $\sup _{n \leq 4 N+b+2}\left\|y^{n}\left(g_{T}^{ \pm}-g\right)\right\|_{L^{1}} \leq e^{-\beta T} \sup _{n \leq 4 N+b+2}\left\|y^{n} g\right\|_{L^{1}}$ for some $\beta>0$;

(5) $\left\|g_{T}^{ \pm(\overline{3})}\right\|_{L^{1}} \leq c e^{3 \beta T}\|g\|_{L^{1}}$.

These can be done by a convolution argument. By $(1), \pi_{g_{T}^{-}}(T, \alpha) \leq \pi_{g}(T, \alpha) \leq$ $\pi_{g_{T}^{+}}(T, \alpha)$. So

$$
\left|\pi_{g}(T, \alpha)-\pi_{g_{T}^{+}}(T, \alpha)\right| \leq \pi_{g_{T}^{+}}(T, \alpha)-\pi_{g_{T}^{-}}(T, \alpha) .
$$

By the Remark (2) of Section 5 the term $O\left(T^{-(N+1)}\right)$ is bounded by

$$
C \sup _{n \leq 4 N+b+2}\left\|y^{n} g\right\|_{L^{1}} T^{-(N+1)}+c\left\|g^{(3)}\right\|_{L^{1}} e^{-\epsilon T} .
$$

In this case,

$$
C \sup _{n \leq 4 N+b+2}\left\|y^{n} g\right\|_{L^{1}}=\sup _{n \leq 4 N+b+2} \int_{-T^{\frac{1}{4 N+b+3}}}^{0}|y|^{n} d y \leq C T .
$$

By Proposition 3, we have that

$$
\begin{aligned}
& \left|\pi_{g_{T}^{ \pm}}(T, \alpha)-\sum_{j=0}^{N+b+2} \frac{1}{T^{j+1}} \int_{V_{0}} e^{-2 \pi i\langle v, \alpha\rangle} \frac{d^{j} \widehat{g}_{T}^{ \pm}}{d s^{j}}(-i \beta(i v)) e^{\beta(i v) T} d v\right| \\
& \quad \leq \frac{c\left\|y^{N+b+2} g_{T}^{ \pm}\right\|_{L^{1}}}{T^{N+b+2}} e^{T h}+c\left(\left\|g_{T}^{ \pm(3)}\right\|_{L^{1}}+\left\|g_{T}^{ \pm}\right\|_{L^{1}}+\cdots+\left\|y^{N+b+2} g_{T}^{ \pm}\right\|_{L^{1}}\right) e^{T(h-\epsilon)} \\
& \quad \leq c_{0} \frac{e^{T h}}{T^{N+b+1}}+c_{1} e^{T(h-\epsilon)}+c_{2} e^{T(h-\epsilon+3 \beta)}
\end{aligned}
$$


By (4),

$$
\sum_{j=0}^{N+b+2} \frac{1}{T^{j+1}}\left|\int_{V_{0}} e^{-2 \pi i\langle v, \alpha\rangle}\left(\frac{d^{j} \widehat{g_{T}^{+}}}{d s^{j}}-\frac{d^{j} \widehat{g}}{d s^{j}}\right)(-i \beta(i v)) e^{\beta(i v) T} d v\right| \leq c_{3} e^{(h-\beta) T} .
$$

Hence,

$$
\begin{aligned}
& \pi_{g}(T, \alpha)-\sum_{j=0}^{N+b+2} \frac{1}{T^{j+1}} \int_{V_{0}} e^{-2 \pi i\langle v, \alpha\rangle} \frac{d^{j} \widehat{g}}{d s^{j}}(-i \beta(i v)) e^{\beta(i v) T} d v \\
& \leq \pi_{g_{T}^{+}}(T, \alpha)-\sum_{j=0}^{N+b+2} \frac{1}{T^{j+1}} \int_{V_{0}} e^{-2 \pi i\langle v, \alpha\rangle} \frac{d^{j} \widehat{g}}{d s^{j}}(-i \beta(i v)) e^{\beta(i v) T} d v \\
& \leq \pi_{g_{T}^{+}}(T, \alpha)-\sum_{j=0}^{N+b+2} \frac{1}{T^{j+1}} \int_{V_{0}} e^{-2 \pi i\langle v, \alpha\rangle} \frac{d^{j} \widehat{g_{T}^{+}}}{d s^{j}}(-i \beta(i v)) e^{\beta(i v) T} d v \\
& \quad+\sum_{j=0}^{N+b+2} \frac{1}{T^{j+1}}\left|\int_{V_{0}} e^{-2 \pi i\langle v, \alpha\rangle}\left(\frac{d^{j} \widehat{g_{T}^{+}}}{d s^{j}}-\frac{d^{j} \widehat{g}}{d s^{j}}\right)(-i \beta(i v)) e^{\beta(i v) T} d v\right| \\
& \leq c_{0} \frac{e^{T h}}{T^{N+b+1}}+c_{1} e^{T(h-\epsilon)}+c_{2} e^{T(h-\epsilon+3 \beta)}+c_{3} e^{T(h-\beta)} .
\end{aligned}
$$

Similarly for $g_{T}^{-}$, we have

$$
\begin{aligned}
& \left|\pi_{g}(T, \alpha)-\sum_{j=0}^{N+b+2} \frac{1}{T^{j+1}} \int_{V_{0}} e^{-2 \pi i\langle v, \alpha\rangle} \frac{d^{j} \widehat{g}}{d s^{j}}(-i \beta(i v)) e^{\beta(i v) T} d v\right| \\
& \leq c_{0} \frac{e^{T h}}{T^{N+b+1}}+c_{1} e^{T(h-\epsilon)}+c_{2} e^{T(h-\epsilon+3 \beta)}+c_{3} e^{T(h-\beta)} .
\end{aligned}
$$

Choosing $\beta<\epsilon / 3$, we have

$$
\left|\pi_{g}(T, \alpha)-\sum_{j=0}^{N+b+2} \frac{1}{T^{j+1}} \int_{V_{0}} e^{-2 \pi i\langle v, \alpha\rangle} \frac{d^{j} \widehat{g}}{d s^{j}}(-i \beta(i v)) e^{\beta(i v) T} d v\right| \leq c \frac{e^{T h}}{T^{N+b+1}} .
$$

We do the same as in Section 4 for $\int_{V_{0}} e^{-2 \pi i\langle v, \alpha\rangle} \frac{d^{\prime} \widehat{g}}{d s^{j}}(-i \beta(i v)) e^{\beta(i v) T} d v$ and obtained

$$
\begin{gathered}
\sum_{j=0}^{N+b+2} \frac{1}{T^{j+1}} \int_{V_{0}} e^{-2 \pi i\langle v, \alpha\rangle} \frac{d^{j} \widehat{g}}{d s^{j}}(-i \beta(i v)) e^{\beta(i v) T} d v \\
=\frac{e^{T h}}{T^{\frac{b}{2}+1}}\left(c_{0}+\sum_{k=1}^{N-1} \frac{c_{k, T}(\alpha)}{T^{k}}+O\left(\frac{1}{T^{N}}\right)\right) .
\end{gathered}
$$

Since for $g=\chi_{\left[-T^{\frac{1}{4 N+b+3}}, 0\right]}, \hat{g}(-i \beta(i v))=g(v)+O\left(e^{-T^{\frac{1}{4 N+b+3}} h}\right)$,

$$
c_{k, T}(\alpha)=c_{k}(\alpha)+O\left(e^{T h-T^{\frac{1}{4 N+b+3}} h}\right) .
$$


Hence

$$
\pi_{g}(T, \alpha)=\frac{e^{T h}}{T^{b / 2+1}}\left(c_{0}+\sum_{k=1}^{N-1} \frac{c_{k}(\alpha)}{T^{k}}+O\left(\frac{1}{T^{N}}\right)\right) .
$$

Now

$$
\pi\left(T-T^{\frac{1}{4 N+b+3}}, \alpha\right)=O\left(e^{T h-T^{\frac{1}{4 N+b+3}} h}\right)
$$

and

$$
\pi\left(T-T^{\frac{1}{4 N+b+3}}, \alpha\right)+\pi_{\chi_{\left[-T^{\left.\frac{1}{4 N+b+3}, 0\right]}\right.}}(T, \alpha)=\pi(T, \alpha) .
$$

However, $e^{-h T^{1 / 4 N+b+3}} \rightarrow 0$ faster than $1 / T^{n}$ for any $n$. So we have the following theorem.

THEOREM 2. Let $M$ be a compact Riemannian manifold with first Betti number $b>0$ and with negative section curvature. Furthermore suppose that either $\operatorname{dim} M=2$ or the sectional curvatures lie between -4 and -1 . For $\alpha=\left(\alpha_{1}, \alpha_{2}, \ldots, \alpha_{b}\right) \in H_{1}(M, \mathbb{Z}) \cong \mathbb{Z}^{b}$, we have

$$
\pi(T, \alpha)=\frac{e^{T h}}{T^{b / 2+1}}\left(c_{0}+\sum_{n=1}^{N} \frac{c_{n}(\alpha)}{T^{n}}+O\left(T^{-(N+1)}\right)\right),
$$

where $c_{0}$ is a constant which is independent of $\alpha$, and

$$
c_{n}(\alpha)=\sum_{l_{1}+l_{2}+\cdots+l_{b}=0}^{2 n} c_{l_{1} l_{2} \ldots l_{b}} \alpha_{1}^{l_{1}} \alpha_{2}^{l_{2}} \ldots \alpha_{b}^{l_{b}}
$$

is a polynomial in $\alpha_{1}, \ldots, \alpha_{b}$ whose degree is $2 n$. Furthermore, $c_{n}(\alpha) \sim(-1)^{n} c\|\alpha\|^{2 n}$, as $\|\alpha\| \rightarrow \infty$.

REMARK. We also can directly obtain $\pi_{\delta}(T, \alpha)$ by taking $g=\chi_{[-\delta, \delta]}$ then using approximation argument.

Now we consider the special case. If we take $N=1$, then (10) reduces

$$
\pi(T, \alpha)=\frac{e^{T h}}{T^{b / 2+1}}\left(c_{0}+\frac{c_{1}(\alpha)}{T}+O\left(T^{-2}\right)\right) .
$$

By the remark following Theorem 1, we have that $c_{0}>0$, and $c_{1}(\alpha)=-\sum_{i, j=1}^{b} c_{i j} \alpha_{i} \alpha_{j}+$ $\sum_{i=1}^{b} b_{i} \alpha_{i}+c$ with

$$
c_{i j}=c \int_{\mathbb{R}^{b}} e^{-\frac{1}{2} \beta^{\prime \prime}(0)(v, v)} v_{i} v_{j} d v \quad(c>0) .
$$

Let $A=\left(c_{i j}\right)_{b \times b}, B=\left(b_{i}\right)_{i=1}^{b}$ We have

$$
\pi(T, \alpha)=\frac{e^{T h}}{T^{b / 2+1}}\left(c_{0}+\frac{-\langle\alpha, A \alpha\rangle+\langle B, \alpha\rangle+c}{T}+O\left(T^{-2}\right)\right) .
$$


Lemma 5. $A$ is positive definite.

Proof. For any $x=\left(x_{1}, x_{2}, \cdots, x_{b}\right)$,

$$
\begin{aligned}
\langle x, A x\rangle & =\sum_{i, j=1}^{b} c_{i j} x_{i} x_{j}=c \sum_{i, j=1}^{b} \int_{\mathbb{R}^{b}} e^{-\frac{1}{2}\left\langle v, \beta^{\prime \prime}(0) v\right\rangle} x_{i} v_{i} x_{j} v_{j} d v \quad(c>0) \\
& =c \int_{\mathbb{R}^{b}} e^{-\frac{1}{2}\left\langle v, \beta^{\prime \prime}(0) v\right\rangle} \sum_{i, j=1}^{b}\left(x_{i} v_{i} x_{j} v_{j}\right) d v=c \int_{\mathbb{R}^{b}} e^{-\frac{1}{2}\left\langle v, \beta^{\prime \prime}(0) v\right\rangle} \sum_{i=1}^{b}\left(x_{i} v_{i}\right)^{2} d v \geq 0 .
\end{aligned}
$$

For a fixed $x \neq 0,\left\{v: \sum_{i=1}^{b} x_{i} v_{i}=0\right\}$ is a hyperplane in $\mathbb{R}^{b}$, so that the integrand is positive almost everywhere. This means that $\langle x, A x\rangle>0$ for $x \neq 0$, hence $A$ is positive definite.

Now we can define $\||x|\|=\langle x, A x\rangle^{1 / 2}$ and \|\|$\cdot \|$ is a norm in $H_{1}(M, \mathbb{R})\left(\right.$ or $\left.\mathbb{R}^{b}\right)$. So we have

$$
\pi(T, \alpha)=\frac{e^{T h}}{T^{b / 2+1}}\left(c_{0}+\frac{-\||| \alpha \mid\|^{2}+\langle B, \alpha\rangle+c}{T}+O\left(T^{-2}\right)\right) .
$$

We note $\pi(T, \alpha)=\pi(T,-\alpha)$ for any $T$. In fact for any $T$, there exists a natural oneto-one correspondence in $\Gamma$ by $\gamma \leftrightarrow-\gamma$. The correspondence preserves the length but reverses the direction, so $\pi(T, \alpha)=\pi(T,-\alpha)$ which implies $B=0$ in (12). So we have the following result.

\section{THEOREM 3.}

$$
\pi(T, \alpha)=\frac{e^{T h}}{T^{b / 2+1}}\left(c_{0}+\frac{c_{1}-\||\alpha|\|^{2}}{T}+O\left(T^{-2}\right)\right) \text { as } T \rightarrow \infty,
$$

where $c_{0}, c_{1}$ are constants and $c_{0}>0$.

It is also easy to see that the following holds.

COROLlaRY 1. For all $\alpha, \beta \in H_{1}(M, \mathbb{Z})$, if $\||\alpha\|||\|||\beta \||$ then, for sufficiently large $T$, we have $\pi(T, \alpha)<\pi(T, \beta)$. In particular, if $\alpha \neq 0$ then, for sufficiently large $T$, $\pi(T, \alpha)<\pi(T, 0)$.

ACKNOWLEDGEMENTS. The author would like to thank Richard Sharp for encouragement and fruitful discussions and is grateful to CVCP and The University of Manchester for financial support.

\section{REFERENCES}

1. N. Anantharaman, Precise counting results for closed orbits of Anosov flows, Ann. Sci. Ećole Norm. Sup. (4) 33 (2000), 33-56.

2. D. Dolgopyat, On decay of correlations in Anosov flows, Ann. of Math. 147 (1998), $357-390$

3. D. Dolgopyat, Prevalence of rapid mixing in hyperbolic flows, Ergod. Th. Dynam. Sys. 18 (1998), 1097-1114.

4. M. Kotani, A note on asymptotic expansions for closed geodesics in homology classes, Math. Ann. (3) 320 (2001), 507-529. 
5. S. Lalley, Closed geodesics in homology classes on surface of variable negative curvature, Duke Math. J. 59 (1989), 795-821.

6. D. Liu, Asymptotic expansion for closed orbits in homology classes for Anosov flows, Math. Proc. Cambridge Philos. Soc. 136 (2004), 383-397.

7. G. Margulis, On some applications of ergodic theory to the study of manifolds of negative curvature, Func. Anal. App. 3 (1969), 89-90.

8. M. Pollicott and R. Sharp, Exponential error terms for growth functions on negatively curved surfaces, Amer. J. Math. 120 (1998), 1019-1042.

9. M. Pollicott and R. Sharp, Asymptotic expansions for closed orbits in homology classes, Geometriae Dedicata 87 (2001), 123-160. 